\title{
Some Cracking Ideas on Egg Incubation
}

\author{
CARINA Edwards ${ }^{1}$, NiCK OVEnden $^{2} \dagger$ and Vivi RotTschäFer ${ }^{3}$ \\ 1 University of Oxford \\ ${ }^{2}$ University College London \\ ${ }^{3}$ University of Leiden
}

(Communicated to MIIR on 6 July 2021)

Study Group: ESGI 46. 31 March - 4 April 2003, bristol

Communicated by: Hilary Ockendon

Industrial Partner: Bristol Zoological Gardens

Presenter: Duncan Bolton

Team Members: Maria Aguareles, Universitat Autonònoma de Barcelona; Paul Dellar, University of Oxford; Jeff Dewynne, University of Oxford; Carina Edwards, University of Oxford; James Healy, University of Southampton; Andrew Hill, University of Bath; Andrew Hogg, University of Bristol; Peter Howell, University of Oxford; Sam Howison, University of Oxford; Katerina Kaouri, University of Oxford; Matthew Dixon, Imperial College; Miranda Lewis, University of Southampton; Nicole Marheineke, Fraunhofer-ITWM, Kaiserslautern; John Ockendon, University of Oxford; Nick Ovenden, University College London; Colin Please, University of Southampton; Tiina Roose, University of Oxford; Vivi Rottschäfer, University of Leiden.

Industrial Sector: Charity

Tools: Lubrication theory, Squeeze films

Key Words: African penguins, Eggs, Incubation, Lubrication theory, Squeeze films.

MSC2020 Codes: 76

\section{Summary}

The preservation of rare and endangered species of birds requires finding efficient methods of breeding them in captivity. How can one ensure that artificial incubation is as successful as natural incubation so that one may breed the species as rapidly as possible? Aside from careful control of temperature and humidity, an important factor to reproduce is that eggs must be turned about their long axis from time to time. In this report we attempt to gain some insight into why eggs have to be turned from a fluid dynamic perspective. Our model suggests the primary reason for turning is to enable mixing in the albumen. This mixing provides the embryo with important nutrients, anti-bacterial agents and disperses waste in the early stages of incubation. Our model also indicates that turning of the egg does not seem to be required for uniform heating, in agreement with practical experience of artificial incubators.

$\dagger$ Corresponding author: n.ovenden@ucl.ac.uk 


\section{Study Group contributors}

Maria Aguareles (Universitat Autonònoma de Barcelona)

Paul Dellar (University of Oxford)

Jeff Dewynne (University of Oxford)

Carina Edwards (University of Oxford)

James Healy (University of Southampton)

Andrew Hill (University of Bath)

Andrew Hogg (University of Bristol)

Peter Howell (University of Oxford)

Sam Howison (University of Oxford)

Katerina Kaouri (University of Oxford)

Matthew Dixon (Imperial College)

Miranda Lewis (University of Southampton)

Nicole Marheineke (Fraunhofer-ITWM, Kaiserslautern)

John Ockendon (University of Oxford)

Nick Ovenden (University College London)

Colin Please (University of Southampton)

Tiina Roose (University of Oxford)

Vivi Rottschäfer (University of Leiden)

\section{Introduction}

This problem was posed by Bristol Zoological Gardens and investigated during the 46th European Study Group with Industry 2003 at the University of Bristol. Bristol Zoo looks after rare and endangered species of birds, one of which is the African penguin, and the Zoo is interested in finding efficient ways of breeding them in captivity. One of the strategies used is the removal of eggs from the nest just after they are laid. If eggs are taken away from the mother, then she will usually lay more. The eggs that are taken away from the penguins then need to be incubated artificially. There are artificial incubation machines in use which attempt to replicate the conditions of natural incubation. The overall goal is thus to ensure that artificial incubation is as successful as natural incubation so that one may breed the species as rapidly as possible.

Three factors are thought to be important for the successful incubation of eggs:

1. Temperature: this must be kept close to the birds' own temperature of $37^{\circ} \mathrm{C}$.

2. Humidity: a successfully hatched egg will typically lose $15 \%-17 \%$ of its mass through vapour loss during the incubation period.

3. Motion: it is necessary for an egg to be rotated from time to time. It is not precisely known why birds do this (there are several theories), but hatching does not occur unless the eggs are subject to some kind of occasional turning. 
One of the questions we tried to answer is whether all of these factors are (equally) important for incubation. In particular, we examined the issue of why eggs need to be turned.

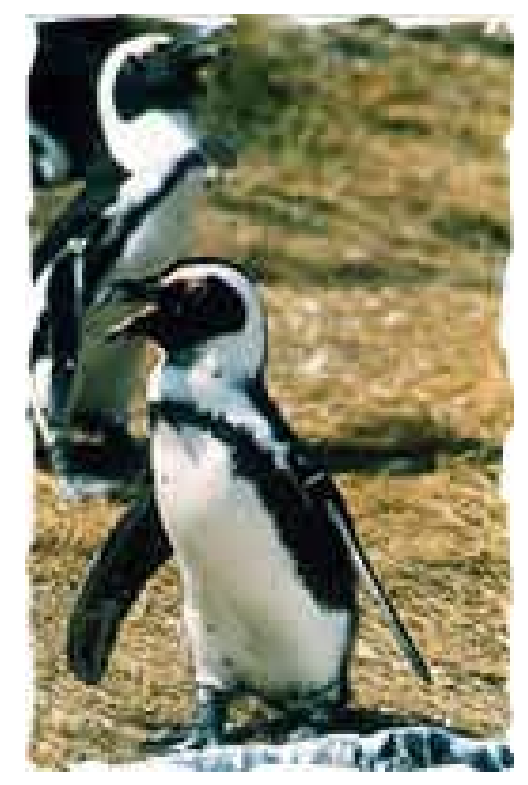

Figure 1: African penguins at Bristol Zoological Gardens.

One possible approach to artificial incubation is to measure in detail how birds incubate their eggs and then try to mimic their behaviour as closely as possible. To this end, a team led by Peter Barham, from the Department of Physics at the University of Bristol, constructed a number of artificial eggs made from nylon which were given to the penguins to incubate. The nylon eggs contained various temperature sensors embedded inside them, together with gravitational switches that could measure orientation. Large data sets of egg temperature and egg rotation were subsequently produced. However, many physical properties of these nylon eggs were (obviously) quite different from those of real ones and, unfortunately as will be noted later in the article, it appeared that the eggs produced too little reliable data to gain any serious insight into the penguins' behaviour.

The main questions that we formulated and tried to answer during the Study Group were the following:

- Why do birds rotate their eggs?

Since there were several theories given in the literature for this, it was the most difficult but also the most important question to answer.

- Does thermal convection play a role during incubation?

- What are the properties of the flow inside the shell following the rotation of the egg?

- Can the results be extended to eggs from different species? 
- How can the nylon eggs be improved?

In this article, we attempt to provide at least partial answers to all of these questions.

\section{$2 \quad$ Egg biology and development}

The eggs of the African penguin are slightly smaller than normal chicken eggs but otherwise appear very similar. Figure 2 contains a sketch of the cross-section of a typical penguin egg during the earliest stages of incubation. The egg contains a yellow spherical yolk, which is covered by a membrane called the yolk sac, surrounded by a transparent visco-elastic fluid called the albumen (egg white). Just after the egg has been laid, the embryo (at this stage called the blastoderm) is a small white spot on the surface of the yolk (at the top). After a couple of days of incubation, the embryo begins to develop inside the yolk sac from this blastoderm.

We will now provide a more detailed description of the yolk and the albumen.

\section{The yolk}

Although not easily seen, the yolk has a layered structure consisting of yellow and white yolk all encased in an inert vitelline membrane. These layers have slightly different

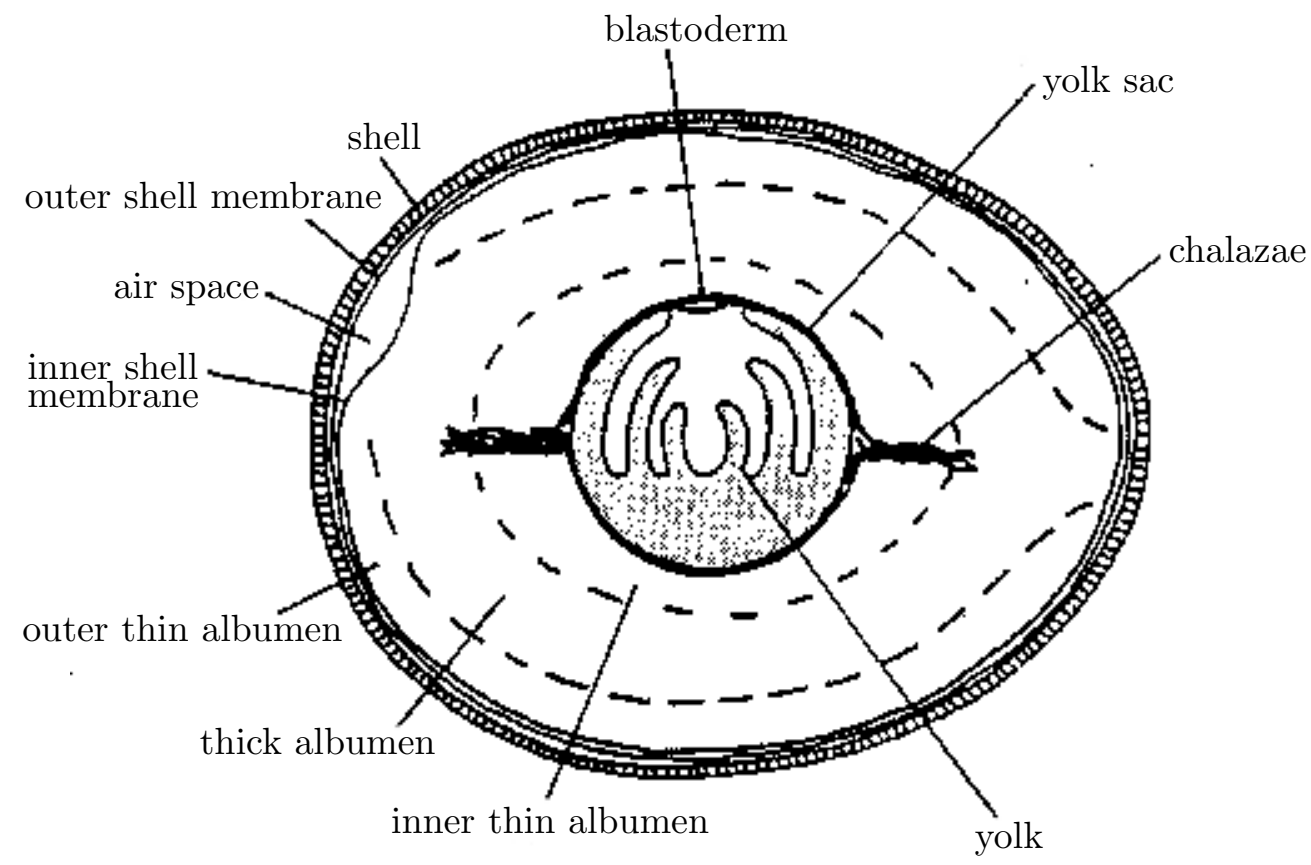

Figure 2: A sketch of the side view of an egg taken along its longest axis. Here the yolk and different regions of albumen are drawn and labelled.

densities leading to stratification on settling. The blastoderm itself is also of a lower 
density than the surrounding yolk and albumen and hence, because the yolk is free to rotate within the albumen, the embryo always returns to the top of the egg after turning.

The yolk contains nutrients (in particular lipids and proteins), vitamins and minerals that are essential for the development of the embryo. During the incubation period the embryo also uses the yolk for the storage of waste products.

\section{The albumen}

The albumen is also a composite structure with a number of distinctive layers surrounding the yolk. The yolk is enclosed and suspended by twisted fibres of albumen proteins called the chalazae (or cords). Immediately adjacent to the yolk is a layer of thin albumen with a thick layer of albumen forming a gel-like capsule surrounding both. There is additionally a further layer of thin albumen adjacent to the shell membrane. The thick albumen has a viscosity of roughly 12 times that of the thin albumen. The albumen contains a variety of proteins that have important anti-microbacterial properties and the albumen also acts as a reservoir of water and ions.

The viscosities and densities of the yolk and the different layers of the albumen can be found in figure 3 where another sketch of a cross section of a penguin egg (this time looking along the egg's major axis) is shown.

\section{The three stages of incubation}

The time taken for an egg to hatch is about 30 days. This incubation period can be divided into three different stages of embryo development that each last approximately one-third of the total incubation time.

\section{Stage 1 (0-10 days)}

In the beginning of this stage the embryo will attach itself to the yolk sac. It will begin to make a vascular system around the yolk sac (remaining inside the yolk); this can be seen in figure 4 where some photos of a chicken egg in Stage 1 are presented. During these first few days the embryo will start to grow and the air space (see figure 2) develops. There is little or no interaction of the contents of the egg with the outside environment since the eggshell structure is initially impermeable (section 4 gives a more detailed explanation why). Experience with artificial incubation suggests that occasional turning of the egg is most crucial at this first stage. In the case that the embryo is still alive at the end of Stage 1, the chance of successful hatching is very high and much less concern needs to be placed on the issue of turning in the later stages.

\section{Stage 2 (10-20 days)}

Much less is known at present about Stages 2 and 3 in comparison to Stage 1. During the second stage the vascular system becomes more extensive and the embryo transforms into a small fetus (figure 5). From this stage onwards there is significant interaction of the egg contents with the outside environment. 


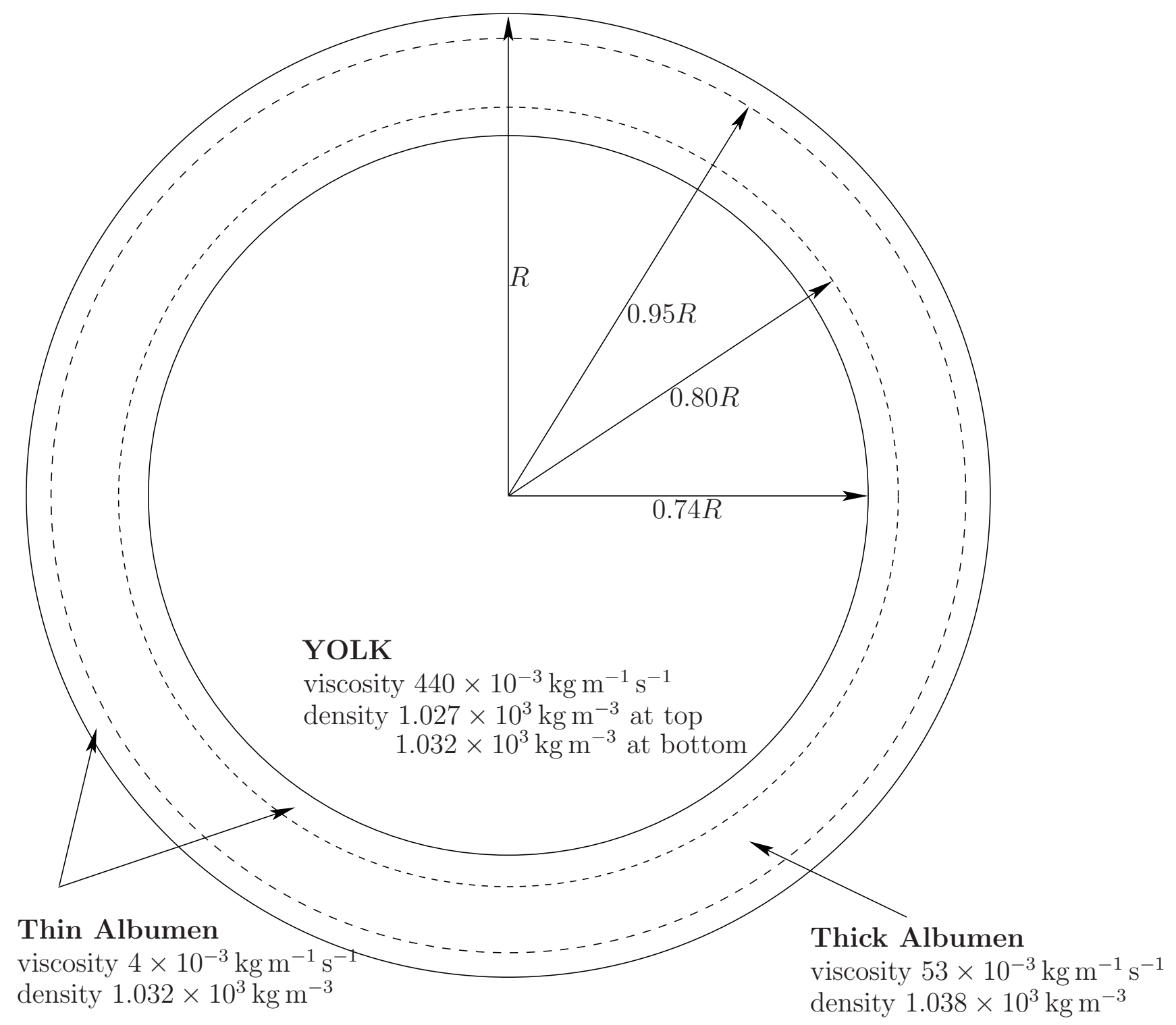

Figure 3: Schematic of a typical penguin egg looking along the egg's major axis. Data is from Romanoff and Romanoff [5]. 

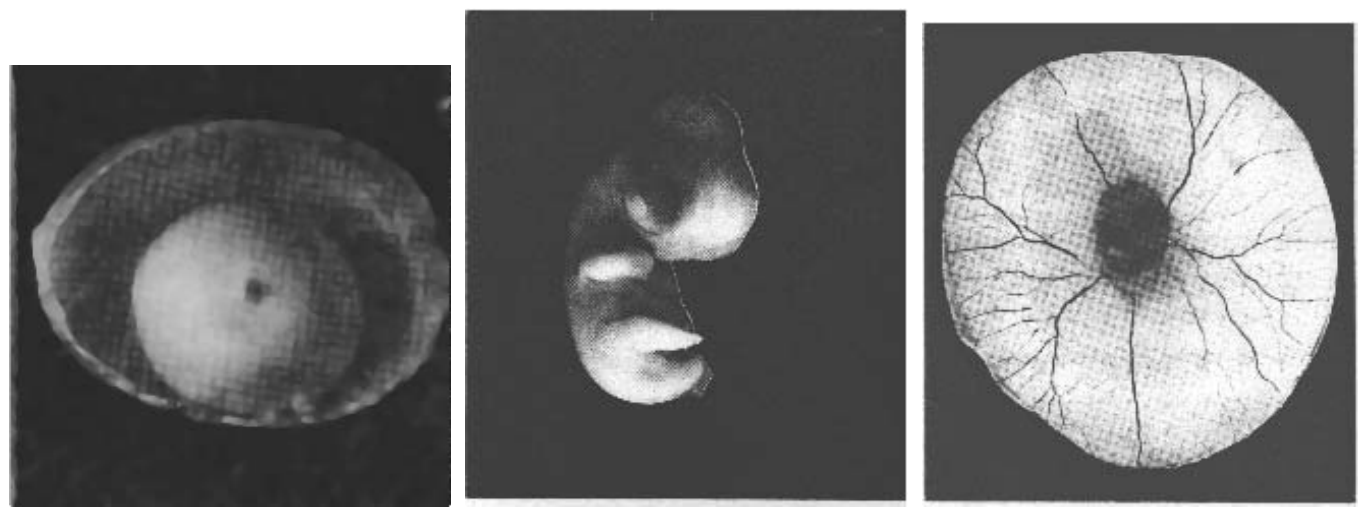

Figure 4: Photos of a chicken egg. One at the start of Stage 1 and two that were taken on the same day at the end of Stage 1.
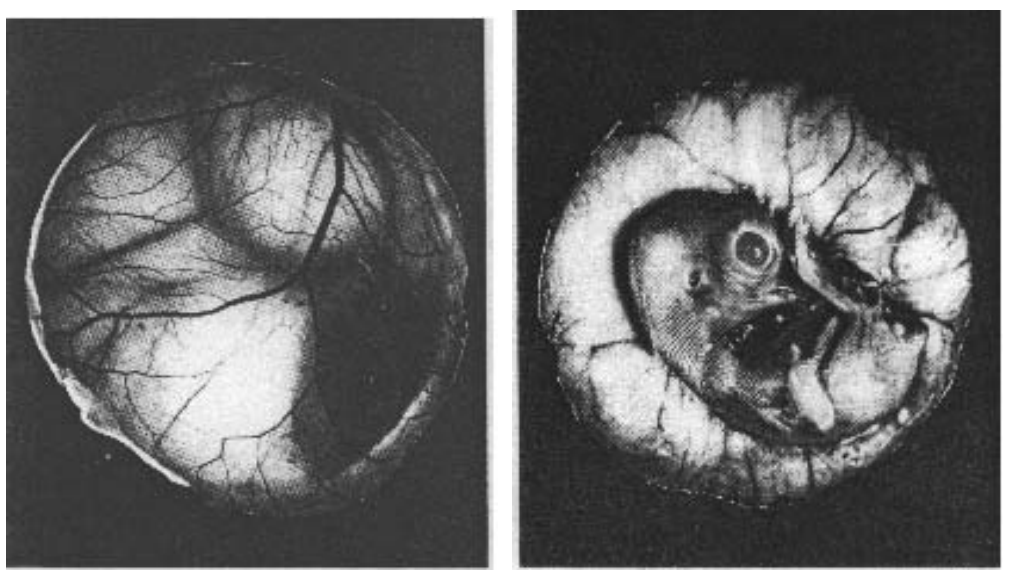

Figure 5: Photos of a chicken egg taken at Stage 2. Both photos were taken on the same day from different viewpoints.

\section{Stage 3 (20-30 days)}

In the final stage of development the fetus will begin to resemble a newly born hatchling which occupies almost the entire space within the shell (figure 6).

Since the turning is most crucial in the first stage, we will from now on focus on that stage (the first couple of days of incubation) only.

\section{The penguin telemetry egg: data analysis}

As we explained briefly in the introduction, an experiment was carried out at Bristol Zoo where nylon eggs were placed in nests of penguins to obtain information on how the birds turn their eggs. This resulted in data for a number of penguins over several days of the incubation period.

The nylon eggs contain several sensors that can measure the temperature and the 

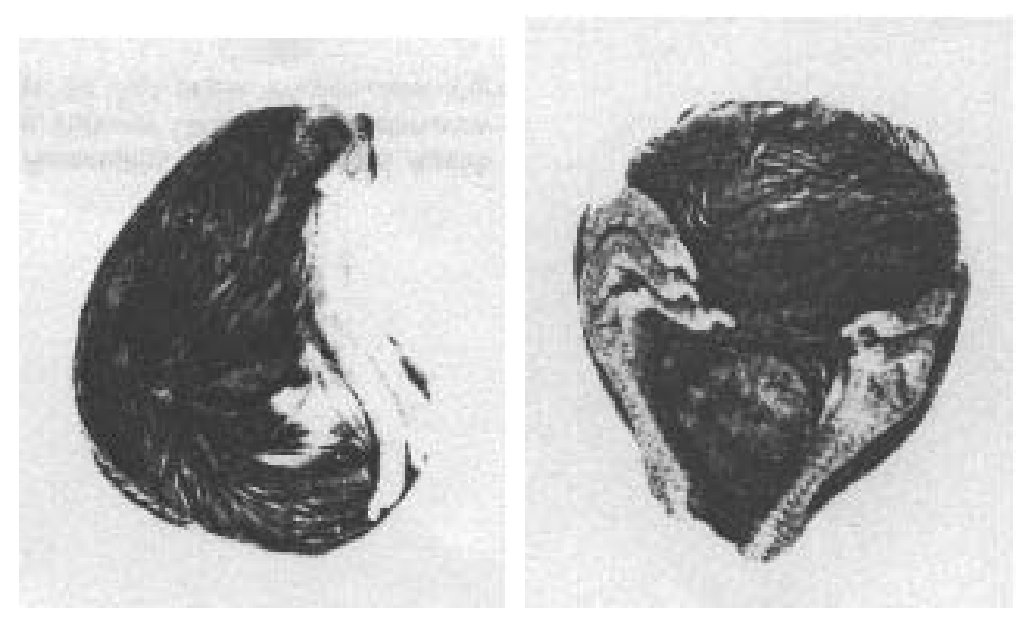

Figure 6: Photos of a chicken inside an egg taken at Stage 3. Both photos were taken on the same day from different viewpoints. In Stage 3 the hatchling takes up most of the space inside the shell.

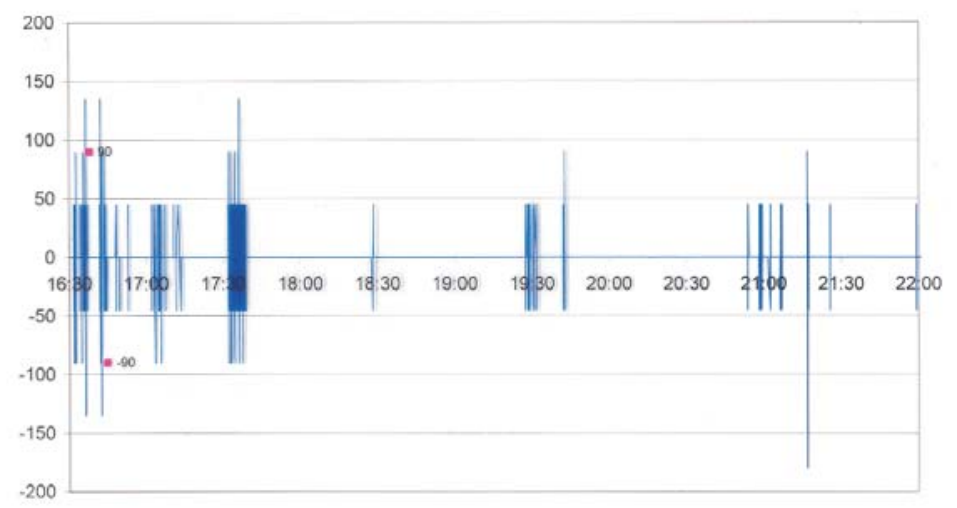

Figure 7: An example of the nylon-egg data: the change of egg angle during penguin incubation over the course of a few hours.

motion of the egg. Inside the egg there are five temperature sensors, four placed around the centre of the egg and one at the tip of the egg. Furthermore, three sensors measure the motion of the egg: two of these are able to give results about the egg's rotation around the longest axis of the egg whereas the third one registers when the egg is flipped over on its longest axis. The usage of only two sensors to measure the angle of rotation resulted in the fact that angles could only be measured in steps of $45^{\circ}$. As a result, rotations from $-22.5^{\circ}$ to $22.5^{\circ}$ were not registered as rotations.

Even though a lot of data was collected from each test, some of this data had to be discarded. For instance, after being placed in the nest the egg had to be heated up to the penguin's body temperature of $37^{\circ} \mathrm{C}$ before the data became relevant. The batteries within the egg would also only last 4-6 hours, and the battery failing would 


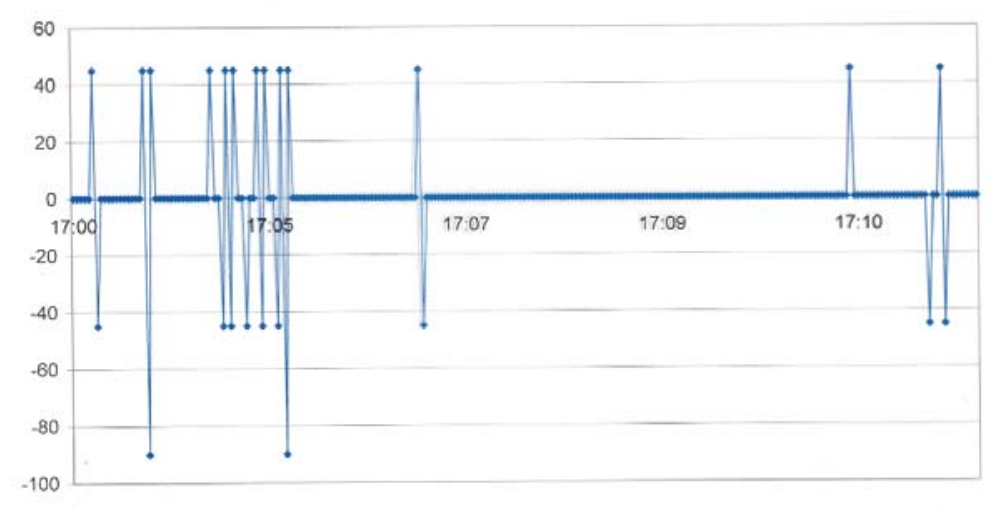

Figure 8: Zooming into a fifteen minute timeframe of data from the previous figure 7. Note that the egg appears to always return to its original position.

be accompanied by the sensors showing an unrealistic temperature rise. As a result, roughly only two hours of usable data could be gained from each data set.

An example of the produced data for egg turning is shown in figures 7 and 8 . The data shows the change in angular position of the egg over the course of the battery life, in steps of $45^{\circ}$, relative to its previous position. We note in the data that there are periods of up to a couple of minutes in which the egg was moved several times. After such a shuffling movement, the egg was then usually not rotated for around 45 minutes or so. This made us curious as to the resulting angle of rotation after each such period of movement. It came as a bit of a surprise to us to find that for most of these periods the resulting angle measured was zero! This is clearly seen in the second figure (figure 8) where we zoom in on a particular fifteen minute time slot. Due to the already-mentioned problems with the current egg design, we do not have much confidence in this conclusion and choose to ignore it and accept observations by penguin-keepers. We await further more accurate experimental evidence in the future.

\section{The Stage 1 turning model: assumptions}

The model is formulated for the first stage of development, which, as discussed in section 2 , is when turning is most crucial. By focussing on this period of development several simplifying assumptions can be made.

1. The egg is a closed system. The eggshell structure, as depicted in figure 9, consists of the cuticle, the true shell, an outer membrane and a fluid-loaded inner membrane. The fluid-loaded inner membrane initially prevents gaseous exchange with the outside world and closes the system. All water vapour loss in the first stage of development can be attributed to losses from this inner membrane. After a few days, the inner membrane loses enough fluid to become porous, enabling gaseous exchange and respiration; this is assumed to occur after the first stage of development. A more detailed description of the changes in shell porosity can be found in Tullett [6]. 


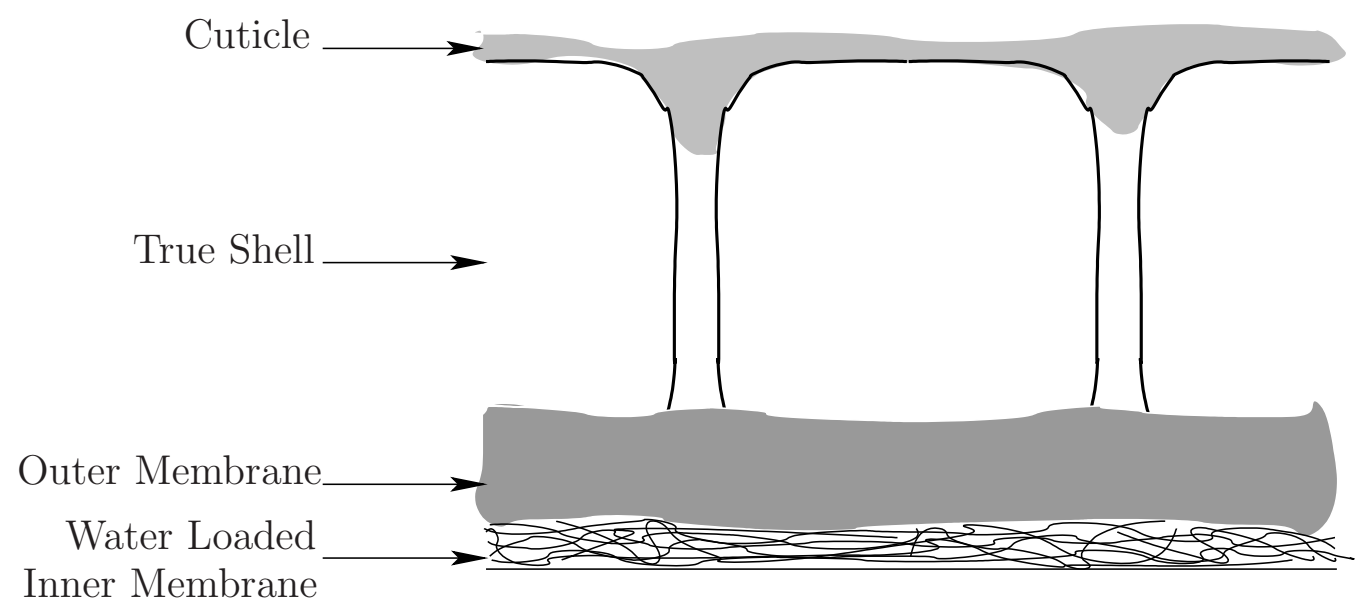

Figure 9: A cross section of the shell illustrating the water loaded membrane.

2. The embryo lies just inside the yolk sac attached to the membrane. It is less dense than the material forming the yolk and is assumed to be physically small during the first stage of development. The buoyancy force of the embryo is assumed to act at a point and returns the embryo to the top of the egg. The slight density stratification of the yolk described in figure 3 may contribute to this process but is not modelled separately.

3. The role of the cords (chalazae) as described in section 2 is unclear. It is initially assumed that the tension in the cords is negligible and thus they are not included in our model. However, we show later that their addition requires only a small modification to the governing equations.

4. The albumen is a viscoelastic fluid. The rotation of the egg by the penguin happens over a short enough timescale that it is assumed to be impulsive. Thus, the albumen can be expected to undergo rigid body rotation. The relaxation of the embryo back to its rest position at the top of the egg happens over a much longer timescale, during which the albumen flows as a viscous liquid. The flow is driven by the buoyancy force of the embryo within the yolk, and additionally the buoyancy of the yolk within the albumen.

5. Where certain physical properties of the albumen and yolk have been difficult to ascertain (such as diffusivity constants), the respective values for pure water have been used, as detailed in Batchelor [1].

\section{Fluid dynamics of the egg: what is the reason for turning?}

It would appear that penguins turn their eggs through a significant angle at intervals of approximately 20 minutes. The hypothesis for this behaviour is that the penguin is redistributing either heat or nutrients within the egg. 


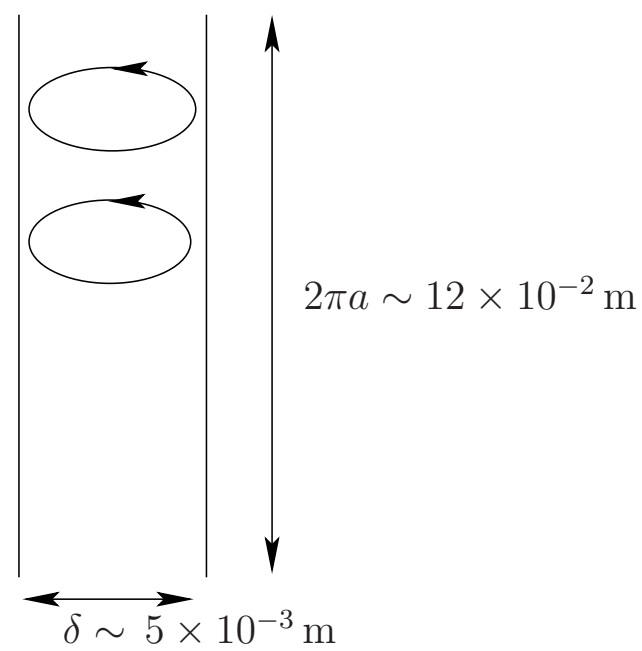

Figure 10: Thermal convection in the albumen annulus.

\subsection{Is convection important?}

As penguins sit on top of their eggs, the system is convectively stable (as heat rises!) and we can conclude that heat transport through the egg is a diffusive process. This can be further confirmed by evaluating the Rayleigh number, which quantifies the relative sizes of the viscous and thermal buoyancy effects. Assuming that any convection present will occur in the albumen annulus surrounding the yolk, the dimensional configuration is that of figure 10. The buoyancy force is of $\mathcal{O}(\rho \alpha g \Delta T)$ per unit volume where $\rho \sim 10^{3} \mathrm{kgm}^{-3}$ is the albumen density, $\alpha \sim 10^{-4} \mathrm{~K}^{-1}$ is the coefficient of thermal expansion (taken for pure water at $\left.20^{\circ} \mathrm{C}\right), g=10 \mathrm{~ms}^{-1}$ is the gravitational acceleration and $\Delta T$ is the temperature difference between shell and yolk. In comparison, the viscous forces are of $\mathcal{O}\left(\mu U / \delta^{2}\right)$ for flow speed $\mathcal{O}(U)$ per unit volume, where $\mu \sim 5 \times 10^{-2} \mathrm{kgm}^{-1} \mathrm{~s}^{-1}$ is the viscosity of the thicker albumen, and $\delta$ is the albumen thickness. Taking $U=2 \pi R / t$ with $t=\delta^{2} / \kappa$, where $R \sim 2 \times 10^{-2} \mathrm{~m}$ is the radius of the egg and $\kappa$ is the thermal diffusivity, gives the Rayleigh number as

$$
\mathbf{R a}=\frac{\alpha \Delta T g \delta^{4}}{2 \pi R \nu \kappa}
$$

The thermal conductivity of the albumen is estimated to be similar to that of water, $k \sim 0.5 \mathrm{~W} \mathrm{~m}^{-1} \mathrm{~K}^{-1}$, with density and specific heat capacity $\rho \sim 10^{3} \mathrm{~kg} \mathrm{~m}^{-3}$ and $C_{p} \sim 3 \times 10^{3} \mathrm{~J} \mathrm{~kg}^{-1} \mathrm{~K}^{-1}$ (also taken to be equal to that of pure water). Thus the thermal diffusivity is estimated to be $\kappa \sim 1 / 6 \times 10^{-6} \mathrm{~m}^{2} \mathrm{~s}^{-1}$. From these values, we find that for a given temperature difference $\Delta T$

$$
\mathbf{R a} \sim \Delta T
$$

As the critical Rayleigh number is of $\mathcal{O}(1000)$, even if the penguin were to heat the egg from below, it would be unable to generate enough heat to set up convection currents. A similar result is obtainable for both the thin albumen layer (treated as a separate entity) and the yolk, leading to the conclusion that convection is negligible everywhere inside the egg. 


\subsection{Thermal diffusion}

The timescale for thermal diffusion is given by $\tau_{\mathcal{D}}=R^{2} / \kappa$. Using the estimates for $R$ and $\kappa$ given above, we find

$$
\tau_{\mathcal{D}} \sim 10^{2}-10^{3} \mathrm{~s}
$$

As the penguin rotates its eggs at intervals of 20 minutes we can take the egg rotation time scale as $\tau_{\mathcal{R}} \sim 10^{3} \mathrm{~s}$. If the penguin needed to turn the egg to maintain a uniform temperature throughout the egg, then we would expect to see $\tau_{\mathcal{D}} \gg \tau_{\mathcal{R}}$. However, it would seem that this is not the case, and so temperature control is not the reason for turning. Our conclusion is backed up by experience from artificial incubators, where eggs must still be turned even though the temperature is uniform throughout. This practical argument was first noted by Drent and is referenced in Deeming [3].

\subsection{Molecular diffusion}

As mentioned in section 2, the albumen surrounding the embryo contains anti-bacterial chemicals, growth hormones and nutrients. It is essential that the embryo is continually supplied with these. However, when we consider the timescale of molecular diffusion $\tau_{\mathcal{M}}=R^{2} / D$, where $D \sim 10^{-9} \mathrm{~m}^{2} \mathrm{~s}^{-1}$ is the molecular diffusivity (assumed to be equal to that for pure water), we find

$$
\tau_{\mathcal{M}} \sim 10^{5} \mathrm{~s}
$$

As $\tau_{\mathcal{M}} \gg \tau_{\mathcal{R}}$ this indicates that one (if not the only) purpose of turning must be to provide the embryo with fresh nutrients contained within the albumen.

\subsection{Yolk settling}

To justify the assumption that the yolk acts as a solid body within the egg with the buoyancy force acting at a point, it is necessary to check that there is no significant settling effect over the relevant timescales. The yolk consists of roughly a $7 \%$ suspension of fat particles (colloids) [5], which will diffuse and settle under their own weight. The relevant timescale for the settling can be calculated by treating the colloids as spheres falling freely under gravity in a viscous fluid. We can then apply Stokes' law [1] to get the terminal velocity as

$$
V_{S}=\frac{2}{9} \frac{\Delta \rho g a^{2}}{\mu_{y o l k}}
$$

where $\Delta \rho$ is the density difference between the fat particles and the rest of the yolk, $a$ is the size of the colloid particles (diameter) and $\mu_{y o l k}$ is the mean viscosity of the yolk. Given the values $\Delta \rho \sim 10 \mathrm{kgm}^{-3}, a \sim 10^{-5} \mathrm{~m}$ and $\mu_{\text {yolk }} \sim 4 \times 10^{-1} \mathrm{kgm}^{-1} \mathrm{~s}^{-1}$ (400 times that of water) from [5], we find that

$$
V_{S} \sim 10^{-7}-10^{-8} \mathrm{~ms}^{-1} .
$$

Using this velocity as an idea of how fast the particles move under gravity, it clearly would take a yolk of radius $10^{-2} \mathrm{~m}$ about three days to settle. Therefore, the assumption of treating the yolk as a solid body appears to be very reasonable. 


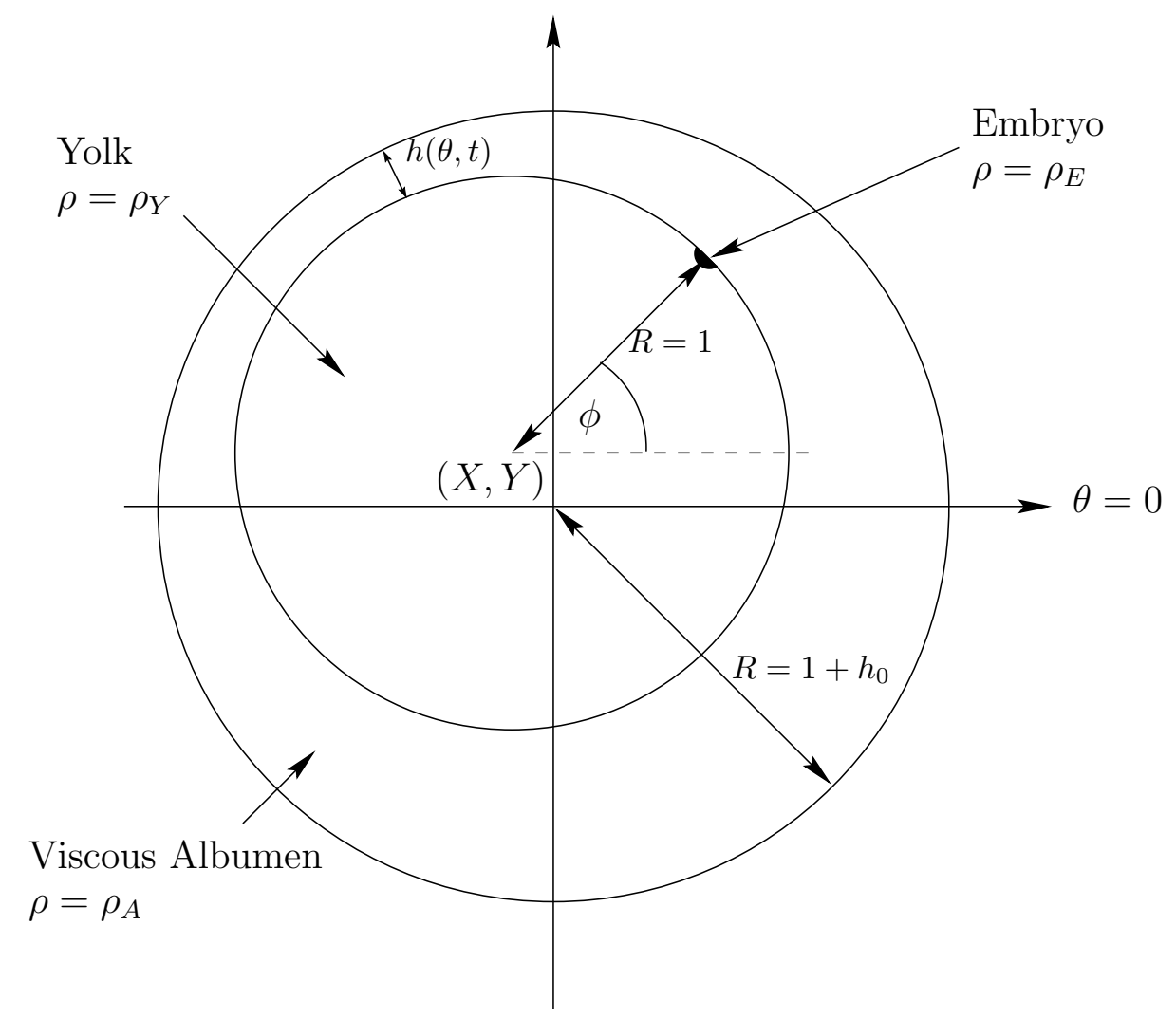

Figure 11: Geometry of egg cross section.

\section{The two-dimensional egg relaxation model (after turning)}

Neglecting the full three-dimensionality of the egg, we first examine the mixing imposed through the action of a buoyant yolk and embryo after an impulsive solid body rotation through some angle.

\subsection{Physical set-up}

After an impulsive solid body rotation, the situation inside our simple two-dimensional egg is presented in figure 11. The figure shows two circles: the outer circle represents the outer shell and the inner circle represents the yolk. The gap inbetween the two circles contains the albumen, and the circles are not concentric due to displacement of the yolk during solid body rotation and buoyancy effects. The embryo is represented by a point of less dense material on yolk's outer membrane at an angle $\phi$ to the horizontal.

All starred variables defined below are dimensional quantities whereas all other variables are non-dimensional. We define a typical length scale to be the radius of the yolk $a^{*}$, and the gap width (albumen thickness) when yolk and shell are concentric is given by $\delta^{*}$. Non-dimensionalising all lengths by $a^{*}$ gives a gap aspect ratio of

$$
h_{0}=\frac{\delta^{*}}{2 \pi a^{*}} .
$$


Defining a Reynolds number based on a typical flow velocity $u^{*}$, yolk radius $a^{*}$ and mean albumen viscosity $\nu^{*}$, lubrication theory applies if the gap aspect ratio $h_{0} \ll 1$ and if the reduced Reynolds number

$$
h_{0}^{2} \operatorname{Re} \ll 1 .
$$

In our model both these conditions shall be assumed to be satisfied.

The densities of embryo, yolk and albumen are given as $\rho_{E}^{*}, \rho_{Y}^{*}$ and $\rho_{A}^{*}$ respectively. Furthermore, we take

$$
\rho_{E}^{*} \leqslant \rho_{Y}^{*} \leqslant \rho_{A}^{*},
$$

leading to the viscous flow being driven by buoyancy forces. Subsequently assuming that pressure, buoyant and viscous forces all balance, we require

$$
\begin{aligned}
\Delta \rho^{*} g^{*}\left(a^{*}\right)^{2} & \sim P^{*} a^{*} \\
\frac{\mu^{*} u^{*}}{\left(\delta^{*}\right)^{2}} & \sim \frac{P^{*}}{a^{*}},
\end{aligned}
$$

where $\Delta \rho^{*}=\rho_{A}^{*}-\rho_{Y}^{*}$ is the typical density difference, $g^{*}$ is gravitational acceleration and $P^{*}$ is the typical pressure generated. Combining these expressions leads to the condition that

$$
\frac{\left(\delta^{*}\right)^{2} \Delta \rho^{*} g^{*}}{\mu^{*} u^{*}} \sim 1
$$

Substituting the values obtained from [5], and shown in figure 3, leads to a typical flow velocity in the albumen $u^{*}$ of $10^{-4} \mathrm{~ms}^{-1}$. Therefore, a typical timescale for the restoring flow after rotation is $a^{*} / u^{*} \sim 10^{2} \mathrm{~s}$.

\section{Gap and squeeze film equation}

The non-dimensional radii of yolk and outer shell are 1 and $1+h_{0}$ respectively. We set the origin of our polar coordinate system at the centre of egg with coordinates $(R, \theta)$. The non-dimensional location of the yolk's centre is at $(R \cos \theta, R \sin \theta)=(X, Y)$ so we have

$$
\left(R_{\text {yolk }}(\theta) \cos \theta-X\right)^{2}+\left(R_{\text {yolk }}(\theta) \sin \theta-Y\right)^{2}=1 .
$$

The outer shell is centred at the origin so

$$
R_{\text {shell }}^{2}(\theta)=\left(1+h_{0}\right)^{2} .
$$

Solving for the gap width $h(\theta)=R_{\text {shell }}-R_{\text {yolk }}$ and assuming $X, Y<h_{0} \ll 1$, we find

$$
h(\theta ; X, Y)=h_{0}-(X \cos \theta+Y \sin \theta)
$$

to first order accuracy.

As the gap and reduced Reynolds number defined above are both taken to be small, the flow in the viscous albumen is predominately one-dimensional in the azimuthal direction with non-dimensional velocity $v_{\theta}(r, \theta)$. The squeeze film equation relating the pressure $P(\theta, t)$ and the gap $h(\theta, t)$ is given by [4]

$$
\frac{\partial}{\partial \theta}\left(\frac{h^{3}}{12} \frac{\partial P}{\partial \theta}\right)=\frac{\dot{\phi}}{2} \frac{\partial h}{\partial \theta}+\frac{\partial h}{\partial t},
$$


where periodicity must be satisfied by means of $h(0, t)=h(2 \pi, t)$ and $P(0, t)=P(2 \pi, t)$.

Defining $r=R-1$, the azimuthal velocity profile is given by

$$
v_{\theta}=\frac{1}{2} \frac{\partial P}{\partial \theta} r(r-h)+\frac{(r-h) \dot{\phi}(t)}{h}
$$

which satisfies the no-slip boundary conditions

$$
\begin{aligned}
& v_{\theta}=\dot{\phi}(t) \quad \text { on } r=0, \\
& v_{\theta}=0 \quad \text { on } r=h .
\end{aligned}
$$

Furthermore, the smaller $\mathcal{O}\left(h_{0}\right)$ radial velocity component $v_{r}$ satisfies,

$$
\begin{array}{ll}
v_{r}=0 & \text { on } r=0, \\
v_{r}=\dot{h} & \text { on } r=h,
\end{array}
$$

as required.

\section{Force balance on the embryo}

The less dense embryo imposes a buoyancy moment on the yolk to spin itself back to the top of the yolk. As inertia is neglected in the lubrication approximation, this buoyancy moment is opposed only by the skin friction generated by the viscous albumen (any torsion generated by the cords attached to the yolk is neglected at present). Balancing these two effects leads to the dimensional expression

$$
\left(\rho_{Y}^{*}-\rho_{E}^{*}\right) A_{E}^{*} g^{*} a^{*} \cos \phi=-\left.\left(a^{*}\right)^{2} \int_{0}^{2 \pi} \mu^{*} \frac{\partial v_{\theta}^{*}}{\partial r^{*}}\right|_{r^{*}=0} \mathrm{~d} \theta,
$$

where $A_{E}^{*}$ is the area of the embryo which we take to be equal to $a^{*} \delta^{*}$. We can nondimensionalise this expression as before with $r^{*}=\delta^{*} r$ and, by further using (5) and (8), we obtain

$$
\Delta m \cos \phi=\int_{0}^{2 \pi} \frac{\dot{\phi}(t)}{h}+\frac{h}{2} \frac{\partial P}{\partial \theta} \mathrm{d} \theta
$$

with

$$
\Delta m=\frac{\rho_{Y}^{*}-\rho_{E}^{*}}{\rho_{A}^{*}-\rho_{Y}^{*}}
$$

Clearly, $\Delta m$ demonstrates how significant the moment generated by the embryo is compared to the buoyancy forces driving the yolk movement as a whole. These yolk forces are derived below. Of course, for cases where the yolk is neutrally buoyant, the density differences throughout the problem can be non-dimensionalised by $\rho_{Y}^{*}-\rho_{E}^{*}$ instead, leading to $\Delta m=1$. 


\section{Force balance on the yolk}

For a non-neutrally buoyant yolk, the yolk will rise to the top of the egg and this will generate a pressure difference in the albumen around the yolk. Once again, as inertia can be neglected and we ignore the effect of the cords, the pressure acting on the yolk balances the buoyancy force present. Hence, resolving in the vertical direction we find

$$
a^{*} \int_{0}^{2 \pi} P^{*} \sin \theta \mathrm{d} \theta=\Delta \rho \pi\left(a^{*}\right)^{2} g^{*} .
$$

Non-dimensionalising using (3) leads to

$$
\int_{0}^{2 \pi} P(\theta, t) \sin \theta \mathrm{d} \theta=\pi
$$

as our driving equation. Similarly, in the horizontal direction there should be no net pressure force on the yolk, thus

$$
\int_{0}^{2 \pi} P(\theta, t) \cos \theta \mathrm{d} \theta=0
$$

\section{Solving the governing equations}

The complete system of governing equations are (6), (7), (14), (16) and (17). Below, they are listed together for clarity:

$$
\begin{aligned}
h(\theta, t) & =h_{0}-[X(t) \cos \theta+Y(t) \sin \theta], \\
\frac{\partial}{\partial \theta}\left(\frac{h^{3}}{12} \frac{\partial P}{\partial \theta}\right) & =\frac{\dot{\phi}}{2} \frac{\partial h}{\partial \theta}+\frac{\partial h}{\partial t} \\
\Delta m \cos \phi & =\int_{0}^{2 \pi} \frac{\dot{\phi}(t)}{h}+\frac{h}{2} \frac{\partial P}{\partial \theta} \mathrm{d} \theta, \\
\int_{0}^{2 \pi} P(\theta, t) \sin \theta \mathrm{d} \theta & =\pi \\
\int_{0}^{2 \pi} P(\theta, t) \cos \theta \mathrm{d} \theta & =0 .
\end{aligned}
$$

Given initial conditions on the position of the yolk and embryo, the system is closed with five equations for five unknowns: $h(\theta, t), X(t), Y(t), \phi(t)$ and $P(\theta, t)$.

Because of the relatively simple gap geometry given by (18), it is possible to substitute this expression for $h(\theta, t)$ into (19) and integrate twice to obtain the pressure,

$$
P(\theta, t)=\int \frac{6 \dot{\phi}\left[h_{0}-X(t) \cos \theta-Y(t) \sin \theta\right]-12 \dot{X}(t) \sin \theta+12 \dot{Y}(t) \cos \theta}{\left[h_{0}-X(t) \cos \theta-Y(t) \sin \theta\right]^{3}} \mathrm{~d} \theta .
$$

Here, both constants of integration can be ignored as (i) an additional uniform pressure gradient $\mathrm{d} P / \mathrm{d} \theta$ would be unrealistic (it would constitute perpetual motion) and (ii) 
a non-spatially varying addition to the pressure would not affect the yolk or embryo dynamics. This expression for the pressure can then be substituted into the remaining three equations (20), (21) and (22) to obtain three coupled ordinary differential equations

$$
\begin{aligned}
\dot{\phi}(t) & =f_{0}(X, Y, \phi), \\
\dot{X}(t) & =f_{1}(X, Y, \phi), \\
\dot{Y}(t) & =f_{2}(X, Y, \phi),
\end{aligned}
$$

for the position of the yolk $(X, Y)$ and embryo $\phi$ inside the egg at all times. For the general case, these coupled o.d.e.s can be solved using standard numerical techniques, given the initial positions of the yolk and embryo at time $t=0$.

\section{Special cases}

In a few cases, an analytical solution to the egg-turning model can be obtained. Two examples are presented below.

\section{Neutrally buoyant yolk with embryo spin-up}

For a neutrally buoyant yolk, $\rho_{A}^{*}=\rho_{Y}^{*}$, the yolk force equations (21) and (22) become irrelevant. The density differences must now be non-dimensionalised with $\rho_{Y}^{*}-\rho_{E}^{*}$ leading to $\Delta m=1$ in (20). Taking the yolk to be positioned in the centre of the egg $(X, Y)=(0,0)$, we have $h(\theta, t)=h_{0}$ from (18) and the squeeze-film equation (19) just leads to $\mathrm{d} P / \mathrm{d} \theta=0$. From (20) we obtain the governing equation

$$
\cos \phi(t)=\frac{2 \pi \dot{\phi}(t)}{h_{0}} .
$$

Integrating this with the initial condition $\phi=\phi_{0}$ at $t=0$ leads to the solution

$$
t=\frac{2 \pi}{h_{0}} \ln \left[\frac{\sec \phi+\tan \phi}{\sec \phi_{0}+\tan \phi_{0}}\right] .
$$

Note that it takes an infinite time for the embryo to get to the top, where $\phi=\pi / 2$.

\section{Buoyant yolk with no horizontal displacement and no embryo spin}

In this case, we assume that the embryo stays at the top throughout, $\phi(t)=\pi / 2$, and that the yolk has no horizontal displacement, $X=0$. In other words, we are just going to examine the yolk floating to the top of the egg from some initial vertical displacement $Y$. From the squeeze-film equation (19), we find

$$
\frac{\partial P}{\partial \theta}=\frac{12 \dot{Y}(t) \cos \theta}{\left[h_{0}-Y(t) \sin \theta\right]^{3}} .
$$


It is possible to integrate this expression to get $P(\theta, t)$ and substituting this into $(21)$ and (22) produces

$$
\dot{Y}=\frac{\left(h_{0}^{2}-Y^{2}\right)^{3 / 2}}{12 \pi}
$$

as an ordinary differential equation for $Y(t)$. For the initial condition $Y=Y_{0}$ at $t=0$, the solution can be written

$$
(t+c)^{2}=\frac{(12 \pi)^{2} Y^{2}}{h_{0}^{4}\left(h_{0}^{2}-Y^{2}\right)} \quad \text { where } c=\frac{12 \pi Y_{0}}{h_{0}^{2}\left(h_{0}^{2}-Y_{0}^{2}\right)^{1 / 2}} .
$$

Similar to that observed in the previous example, the yolk takes an infinite time for $Y \rightarrow h_{0}$ and thus reach the top.

\section{The role of shearing in improving the diffusion of nutrients and waste products}

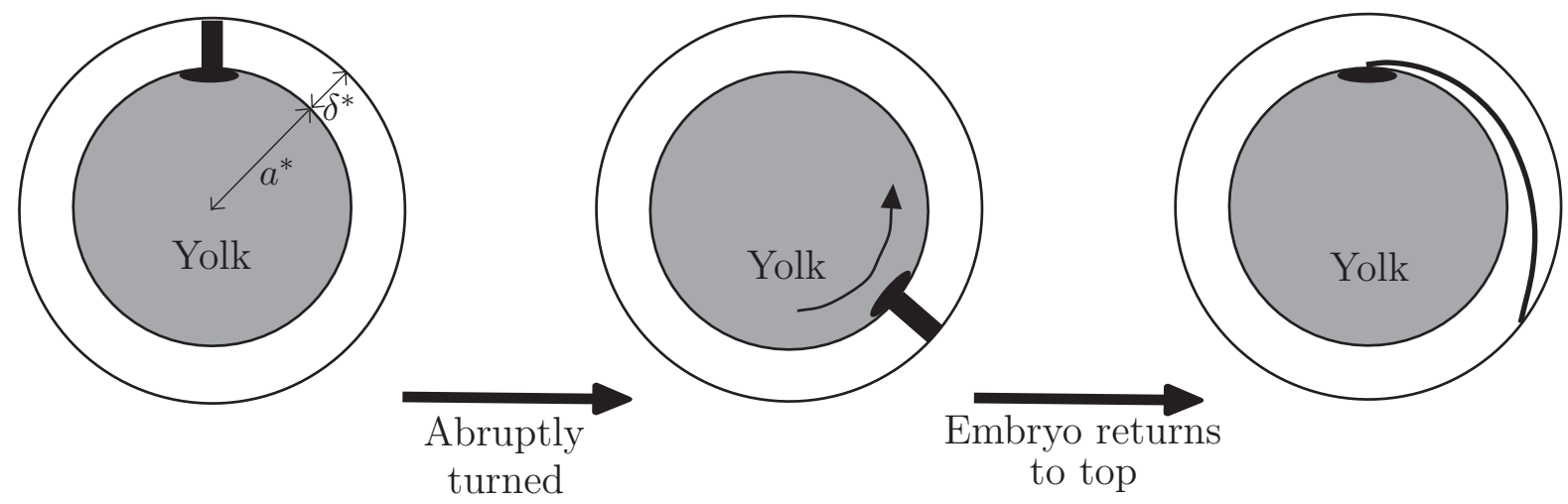

Figure 12: How a shearing flow can help to diffuse waste and nutrients

Here we attempt to explain why the turning of the egg and subsequent spinning of the yolk by the lighter embryo improves access to nutrients and disperses waste effectively. The left-hand diagram of figure 12 shows the inside of an egg with the embryo resting at the top. We assume the radius of the yolk is $a^{*} \sim 10^{-2} \mathrm{~m}$ and that the width of the surrounding albumen layer is $\delta^{*} \sim 10^{-3} \mathrm{~m}$. After some time at rest, the embryo expends the nutrients in the neighbouring albumen, leaving a strip (marked in black) of albumen that has become full of waste products and devoid of nutrients. Assuming this strip to be of size $\delta^{*} \times \delta^{*}$ and the molecular diffusion coefficient to be $D \sim 10^{-9} \mathrm{~m}^{2} \mathrm{~s}^{-1}$ (as given in section 5.3) then the time taken to for this strip to diffuse away will be of the order

$$
\tau \sim \frac{\delta^{* 2}}{D} \sim 10^{3} \mathrm{~s}
$$

The following two diagrams in figure 12, show what happens to this strip when the egg is turned. Following an impulsive rotation about some angle, the lighter embryo then 
forces the yolk to spin round until the embryo is back at the top of the egg. Given an $\mathcal{O}(1)$ turning angle, it is clear that the shearing flow in the albumen stretches the length of the strip by a factor $a^{*} / \delta^{*} \sim 10$. Similarly, the width of the strip $w$ reduces by the same factor $10^{-1}$, leading to a strip now only of width $w \sim 10^{-4} \mathrm{~m}$. The timescale of the diffusion process is proportional to $w^{* 2}$, meaning that the stretched strip only takes

$$
\tau \sim \frac{w^{* 2}}{D} \sim 10 \mathrm{~s}
$$

to diffuse. Hence, the embryo's access to nutrients is greatly increased by occasional turning.

\section{Conclusions}

An examination of the egg turning from a fluid dynamics perspective appears to suggest that the reason for turning is to enable mixing in the albumen. This mixing provides the embryo with important nutrients, anti-bacterial agents and disperses waste in the early stages of incubation. Turning of the egg does not seem to be required for uniform heating, an argument borne out not only by our calculations but from practical experience with artificial incubators.

The fluid flow inside the egg during turning can be described in the following way. No convection currents occur inside the egg during heating by the mother penguin, so the fluid remains initially at rest with the buoyant embryo and buoyant yolk at the top of the egg. An impulsive turn by the penguin induces a solid body rotation of the egg contents by some angle. Slow viscous flow in the albumen is subsequently generated by buoyancy forces, as the embryo and yolk rise back up to the top of the egg. This appears to induce mixing in the albumen which provides the embryo with greater access to nutrients and anti-bacterial agents. The viscous flow takes a typical time of $10^{2} \mathrm{~s}$ with turning occurring every $10^{3} \mathrm{~s}$.

Of course, the model provided can be applied to different species of egg. The different length scales (of yolk/shell size ratio) and yolk, albumen and embryo densities will lead to different non-dimensional parameters (aspect ratio $h_{0}$ and $\Delta m$ ), which means that the turning times and habits of different species of birds can be analysed and contrasted. On the whole, this examination of the fluid flow within the egg should provide developers of artificial incubators with some further guidance to how eggs should be turned.

Apart from naturally the short time the group had to investigate this problem, the lack of reliable information on the penguins' turning habits from the nylon egg experiments prevents any further development towards a more accurate model. We would recommend that any new artificial eggs possess a longer battery life and provide improved measurement of the egg's exact position and orientation at all times. In this way, a simple animation of the egg could be reproduced on a computer for detailed examination. Finally, to improve and validate our model for a real incubator, more rheological and density measurements of the albumen and yolk are needed, as well as a better idea of the strength and purpose of the chalazae (cords) which attach themselves to the yolk sac. 


\section{Extensions to the egg relaxation model}

Of course, the model presented is highly simplified, although it does appear to contain the necessary physics to explain why eggs are turned. Possible improvements and extensions to the model are listed below.

\section{Three-dimensional squeeze film}

An obvious more realistic extension would be to develop a squeeze film model in three dimensions, with two spheres representing the outer shell and yolk respectively. In fact, the elongated shape of a real egg along one axis could also be incorporated in the expression for the gap width $h$ in a straightforward manner.

\section{Include cord tension/torsion}

The effect of the cords (or chalazae), as described in the introduction, can easily be incorporated into our current model should they prove significant. For instance, the effect of cord tension on the yolk position can be simply modelled by including the terms $-K Y$ and $-K X$ (for some empirically determined constant $K$ ) in the vertical force balance (21) and horizontal force balance (22) respectively. Similarly, the effect of torsion caused by the turning of the yolk can also be modelled with a $-K_{2} \phi$ (for some empirically determined constant $K_{2}$ ) term in the embryo moment equation (20).

\section{Viscoelasticity of albumen}

No real attempt has been made to model the complex viscoelastic behaviour of the albumen and we have little justification (other than intuition) that the egg undergoes solid body rotation followed by slow viscous flow driven by buoyancy forces. One possible future approach might be to treat the three layers of albumen (see introduction) separately in a multi-cylinder model, with matching at each interface. Clearly, full viscoelastic modelling requires much greater physical understanding of the behaviour of the albumen than is currently available in the literature.

\section{Non-circular/non-spherical yolk}

The settling of the colloids in the yolk may cause the yolk's shape to be non-circular (or non-spherical). This effect could also be included in the model by some alteration of the gap function $h(\theta, t)$.

\section{Acknowledgements}

We would like to thank Duncan Bolton from Bristol Zoological Gardens for suggesting such an interesting and enjoyable problem for the Study Group. We are also grateful to Peter Barham of the Department of Physics, University of Bristol for providing and explaining the artificial egg data. Of course, many thanks are due to Eddie Wilson and co. for organising such an excellent Study Group. 
Last but not least, we are most grateful to the penguins of Bristol Zoo for being so cooperative and photogenic on our visit during the Study Group week. We wish Bristol Zoo and other similar institutions every success in their valuable attempts to protect endangered species around the world.

\section{References}

[1] Batchelor G.K., An Introduction to Fluid Dynamics, Cambridge University Press, 1967.

[2] Deeming D.C., Principles of Artificial Incubation for Game Birds (a practical guide), Ratite Conference, 2000.

[3] Deeming D.C., Egg Incubation: its Effects on Embryonic Development in Birds and Reptiles 307-323, 1982.

[4] Ockendon H. \& Ockendon J.R., Viscous Flow, 1995.

[5] Romanoff A.L.\& Romanoff A.J.S., The Avian Egg, J.Wiley and Sons Inc., New York, 1949.

[6] Tullett S.G., Minireview: the Porosity of Avian Eggshells, Comp. Biochem. Physiol. 78A 5-13, 1984. 